\title{
Some Inequalities of Extended Hypergeometric Functions
}

\author{
Shilpi Jain ${ }^{1}\left(\mathbb{D}\right.$, Rahul Goyal $^{2}(\mathbb{D})$ and Praveen Agarwal ${ }^{2,3,4}\left(\mathbb{D}\right.$ and Juan L. G. Guirao ${ }^{5,6, *(D)}$ \\ 1 Department of Mathematics, Poornima College of Engineering, Jaipur 302021, India; \\ shilpijain1310@gmail.com \\ 2 Department of Mathematics, Anand International College of Engineering, Jaipur 303012, India; \\ rahul.goyal01@anandice.ac.in (R.G.); praveen.agarwal@anandice.ac.in \\ or goyal.praveen2011@gmail.com (P.A.) \\ 3 Department of Mathematics, Harish-Chandra Research Institute, Allahabad 211019, India \\ 4 International Center for Basic and Applied Sciences, Jaipur 302029, India \\ 5 Departamento de Matematica Aplicada y Estadistica, Universidad Politecnica de Cartagena, \\ Hospital de Marina, 30203 Murcia, Spain \\ 6 Department of Mathematics, Faculty of Science, King Abdulaziz University, P.O. Box 80203, \\ Jeddah 21589, Saudi Arabia \\ * Correspondence: Juan.Garcia@upct.es
}

check for

updates

Citation: Jain, S.; Goyal, R.; Agarwal, P.; Guirao, J.L.G. Some Inequalities of Extended Hypergeometric Functions. Mathematics 2021, 9, 2702. https:// doi.org/10.3390/math9212702

Academic Editor: Yuri Luchko

Received: 23 September 2021

Accepted: 18 October 2021

Published: 25 October 2021

Publisher's Note: MDPI stays neutral with regard to jurisdictional claims in published maps and institutional affiliations.

Copyright: (c) 2021 by the authors. Licensee MDPI, Basel, Switzerland. This article is an open access article distributed under the terms and conditions of the Creative Commons Attribution (CC BY) license (https:// creativecommons.org/licenses/by/ $4.0 /)$.

\begin{abstract}
Hypergeometric functions and their inequalities have found frequent applications in various fields of mathematical sciences. Motivated by the above, we set up certain inequalities including extended type Gauss hypergeometric function and confluent hypergeometric function, respectively, by virtue of Hölder integral inequality and Chebyshev's integral inequality. We also studied the monotonicity, log-concavity, and log-convexity of extended hypergeometric functions, which are derived by using the inequalities on an extended beta function.
\end{abstract}

Keywords: gamma function; classical Euler beta function; Gauss hypergeometric function; confluent hypergeometric function; Mittag-Leffler function; log-convexity; log-concavity

MSC: 33B15; 33C05; 33C15; 33E12

\section{Introduction and Preliminaries}

In mathematics, the theory of special functions is an area that plays a vital role due to its applications in real analysis, functional analysis, geometry, physics, and many more subjects of science. Special functions can be defined by power series, generating functions, infinite products, and other series in orthogonal functions. In the past few years, many researchers and authors have been engaged in working on theory and applications of the special functions [1-8]. Inequalities and extensions are both important topics in the theory of special functions, but from a theoretical point of view, very few inequalities involving hypergeometric functions and extended hypergeometric functions seem to have appeared in the literature until now. Here, we aim to introduce some inequalities of extended hypergeometric functions.

Very recently, Goyal and Jain et al. [9,10] have extended the beta function, Gauss hypergeometric function, confluent hypergeometric function and studied various properties of these extended functions. They also studied the increasing or decreasing nature (monotonicity), log-concavity, and log-convexity of extended beta function in [10].

The extended beta function is defined as [9]:

$$
B_{\left(s_{1}, s_{2}\right)}^{(s)}\left(w_{1}, w_{2}\right)=\int_{0}^{1} t^{w_{1}-1}(1-t)^{w_{2}-1} E_{s_{1}, s_{2}}\left(-r(t(1-t))^{-1}\right) d t,
$$

where $\min \left\{\Re\left(w_{1}\right), \Re\left(w_{2}\right)\right\}>0, r \geq 0, \Re\left(s_{1}\right)>0, \Re\left(s_{2}\right)>0$ and $E_{s_{1}, s_{2}}(z)$ is the 2parameter Mittag-Leffler function. 
The extended Gauss hypergeometric function is defined as [10]:

$$
F_{\left(s_{1}, s_{2}\right)}^{(s)}\left(q_{0}, q_{1}, q_{2} ; z\right)=\sum_{k=0}^{\infty} \frac{B_{\left(s_{1}, s_{2}\right)}^{(s)}\left(q_{1}+k, q_{2}-p_{1}\right)}{B\left(q_{1}, q_{2}-q_{1}\right)}\left(q_{0}\right) \frac{z^{k}}{k !},
$$

where $\Re\left(q_{2}\right)>\Re\left(q_{1}\right)>0, \Re\left(s_{1}\right)>0, \Re\left(s_{2}\right)>0, s \geq 0,|z|<1$ and $B_{\left(s_{1}, s_{2}\right)}^{(s)}\left(w_{1}, w_{2}\right)$ is the extended beta function.

Integral representation of the extended Gauss hypergeometric function is defined as [10]:

$$
\begin{gathered}
F_{\left(s_{1}, s_{2}\right)}^{(s)}\left(q_{0}, q_{1}, q_{2} ; z\right)= \\
\frac{1}{B\left(q_{1}, q_{2}-q_{1}\right)} \int_{0}^{1} t^{q_{1}-1}(1-t)^{q_{2}-q_{1}-1}(1-z t)^{-q_{0}} E_{s_{1}, s_{2}}\left(-s(t(1-t))^{-1}\right) d t
\end{gathered}
$$

where $\left(\Re\left(q_{2}\right)>\Re\left(q_{1}\right)>0, \Re\left(s_{1}\right)>0, \Re\left(s_{2}\right)>0, s \geq 0\right.$ and $\left.|z|<1\right)$.

$$
\Phi_{\left(s_{1}, s_{2}\right)}^{(s)}\left(q_{1}, q_{2} ; z\right)=\sum_{k=0}^{\infty} \frac{B_{\left(s_{1}, s_{2}\right)}^{(s)}\left(q_{1}+k, q_{2}-q_{1}\right)}{B\left(q_{1}, q_{2}-q_{1}\right)} \frac{z^{k}}{k !}
$$

where $\left(\Re\left(q_{2}\right)>\Re\left(q_{1}\right)>0, \Re\left(s_{1}\right)>0, \Re\left(s_{2}\right)>0\right.$ and $\left.s \geq 0\right)$.

Integral representation of the extended confluent hypergeometric function is defined as [10]:

$$
\begin{aligned}
& \Phi_{\left(s_{1}, s_{2}\right)}^{(s)}\left(q_{1}, q_{2} ; z\right)= \\
& \quad \frac{1}{B\left(q_{1}, q_{2}-q_{1}\right)} \int_{0}^{1} t^{q_{1}-1}(1-t)^{q_{2}-q_{1}-1} e^{z t} E_{s_{1}, s_{2}}\left(-s(t(1-t))^{-1}\right) d t
\end{aligned}
$$

where $\left(\Re\left(q_{2}\right)>\Re\left(q_{1}\right)>0, \Re\left(s_{1}\right)>0, \Re\left(s_{2}\right)>0\right.$ and $\left.s \geq 0\right)$.

We also require some important results that were published earlier in [10-17] to obtain our main results.

Theorem 1 ([10]). Assume that:

- $\quad x^{\prime \prime}, y^{\prime \prime}, x_{1}^{\prime \prime}, y_{1}^{\prime \prime}$ are non-zero and non-negative numbers such that $\left(x^{\prime \prime}-x_{1}^{\prime \prime}\right)\left(y^{\prime \prime}-y_{1}^{\prime \prime}\right) \geq 0$,

- $u_{1} \in[0,1]$ and $u_{2} \in[0,1]$.

Then

$$
B_{\left(u_{1}, u_{2}\right)}^{(u)}\left(x^{\prime \prime}, y_{1}^{\prime \prime}\right) \cdot B_{\left(u_{1}, u_{2}\right)}^{(u)}\left(x_{1}^{\prime \prime}, y^{\prime \prime}\right) \leq B_{\left(u_{1}, u_{2}\right)}^{(u)}\left(x^{\prime \prime}, y^{\prime \prime}\right) B_{\left(u_{1}, u_{2}\right)}^{(u)}\left(x_{1}^{\prime \prime}, y_{1}^{\prime \prime}\right)
$$

Theorem 2 ([10]). The map $\left(x^{\prime}, y^{\prime}\right) \longmapsto B_{\left(u_{1}, u_{2}\right)}^{(u)}\left(x^{\prime}, y^{\prime}\right)$ is logarithmically convex on $\mathbb{R}^{+} \times \mathbb{R}^{+}$ $\forall u \geq 0$, with $u_{1} \in[0,1]$ and $u_{2} \in[0,1]$. Moreover:

$$
\left[B_{\left(u_{1}, u_{2}\right)}^{(u)}\left(\frac{x_{1}^{\prime}+x_{2}^{\prime}}{2}, \frac{y_{1}^{\prime}+y_{2}^{\prime}}{2}\right)\right]^{2} \leq B_{\left(u_{1}, u_{2}\right)}^{(u)}\left(x_{1}^{\prime}, y_{1}^{\prime}\right) \cdot B_{\left(u_{1}, u_{2}\right)}^{(u)}\left(x_{2}^{\prime}, y_{2}^{\prime}\right)
$$

Lemma 1 ([11]). consider $f_{1}^{\prime}(y)=\sum_{k=0}^{\infty} a_{k}^{\prime} y^{k}$ and $g_{1}^{\prime}(y)=\sum_{k=0}^{\infty} b_{k}^{\prime} y^{k}$ with $a_{k}^{\prime} \in R$ and $b_{k}^{\prime}>0$ $\forall k$, converges on $(-\beta, \beta)$. Then, if the sequence $\left(\frac{a_{k}^{\prime}}{b_{k}^{\prime}}\right)_{k \geq 0}$ is decreasing (or increasing, respectively), subsequently the mapping $y \mapsto \frac{f_{1}^{\prime}(y)}{g_{1}^{\prime}(y)}$ is also decreasing (or increasing, respectively) on $(0, \beta)$. 
Lemma 2 ([10,12,13] Hölder Inequality). Let $\theta_{1}^{\prime}$ and $\theta_{2}^{\prime}$ be positive numbers such that

$$
\frac{1}{\theta_{1}^{\prime}}+\frac{1}{\theta_{2}^{\prime}}=1
$$

Let $f_{1}^{\prime}, f_{2}^{\prime}:\left[c^{\prime}, d^{\prime}\right] \longrightarrow(-\infty,+\infty)$ be integrable functions. Then,

$$
\int_{c^{\prime}}^{d^{\prime}} f_{1}^{\prime}(x) f_{2}^{\prime}(x) d x \leq\left(\int_{c^{\prime}}^{d^{\prime}} f_{1}^{\prime}(x)^{\theta_{1}^{\prime}} d x\right)^{\frac{1}{\theta_{1}^{\prime}}} \cdot\left(\int_{c^{\prime}}^{d^{\prime}} f_{2}^{\prime}(x)^{\theta_{2}^{\prime}} d x\right)^{\frac{1}{\theta_{2}^{\prime}}} .
$$

Lemma 3 ([10,14-16] Chebyshev's integral inequality). Let $f_{1}^{\prime}, f_{2}^{\prime}:\left[c^{\prime}, d^{\prime}\right] \subseteq(-\infty,+\infty) \longrightarrow$ $(-\infty,+\infty)$ be integrable functions. Assume that:

$$
\left[f_{1}^{\prime}(x)-f_{1}^{\prime}(y)\right]\left[f_{2}^{\prime}(x)-f_{2}^{\prime}(y)\right] \gtreqless 0, \quad \forall x^{\prime}, y^{\prime} \in\left[c^{\prime}, d^{\prime}\right] .
$$

Let $h^{\prime}(x):\left[c^{\prime}, d^{\prime}\right] \subseteq(-\infty,+\infty) \longrightarrow(-\infty,+\infty)$ be a positive integrable function. Then:

$$
\int_{c^{\prime}}^{d^{\prime}} h^{\prime}(x) f_{1}^{\prime}(x) d x \cdot \int_{c^{\prime}}^{d^{\prime}} h^{\prime}(x) f_{2}^{\prime}(x) d x \gtreqless \int_{c^{\prime}}^{d^{\prime}} h^{\prime}(x) d x \cdot \int_{c^{\prime}}^{d^{\prime}} h^{\prime}(x) f_{1}^{\prime}(x) f_{2}^{\prime}(x) d x .
$$

Theorem 3 ([17]). Let

$$
f(b, y)=\sum_{k \geq 0} f_{k}^{\prime} \frac{(b)_{k}}{k !} y^{k}
$$

where, $f_{k}^{\prime}$ is not depend on $b$, and we consider that $c>b>0$ and $\delta>0$, then the mapping $f(b+\delta, y) f(c, y)-f(c+\delta, y) f(b, y)=\sum_{m^{\prime} \geq 2} \phi_{m}^{\prime} y^{m}$ has negative power series coefficient $\phi_{m}^{\prime}<$ 0 , so that $b \mapsto f(b, y)$ is strictly log-convex for $y>0$ if the sequence $\left(\frac{f_{k}^{\prime}}{f_{k-1}^{\prime}}\right)$ is increasing.

\section{Main Results}

In this section, we are introducing some inequalities, monotonicity, log-convexity and log-concavity of the extended confluent hypergeometric function $\Phi_{\left(s_{1}, s_{2}\right)}^{(s)}\left(q_{1}, q_{2} ; x\right)$ and extended Gauss hypergeometric function $F_{\left(s_{1}, s_{2}\right)}^{(s)}\left(q_{0}, q_{1}, q_{2} ; x\right)$, respectively.

2.1. Inequalities of Confluent Hypergeometric Function $\Phi_{\left(s_{1}, s_{2}\right)}^{(s)}\left(q_{1}, q_{2} ; x\right)$

Theorem 4. Consider $q_{1} \geq 0$ and $q_{2}, q_{3}>0$ then for $q_{2}-q_{3} \geq 0$ the mapping

$$
x \mapsto \frac{\Phi_{\left(s_{1}, s_{2}\right)}^{(s)}\left(q_{1}, q_{2} ; x\right)}{\Phi_{\left(s_{1}, s_{2}\right)}^{(s)}\left(q_{1}, q_{3} ; x\right)},
$$

is increasing on $(0, \infty)$.

Proof of Theorem 4. By the definition of the extended confluent hypergeometric function (4), we have:

$$
\frac{\Phi_{\left(s_{1}, s_{2}\right)}^{(s)}\left(q_{1}, q_{2} ; x\right)}{\Phi_{\left(s_{1}, s_{2}\right)}^{(s)}\left(q_{1}, q_{3} ; x\right)}=\frac{\sum_{k=0}^{\infty} \frac{B_{\left(1_{1}, s_{2}\right)}^{(s)}\left(q_{1}+k, q_{2}-q_{1}\right)}{B\left(q_{1}, q_{2}-q_{1}\right)} x^{k}}{\sum_{k=0}^{\infty} \frac{B_{\left(1_{1}, s_{2}\right)}^{(s)}\left(q_{1}+k, q_{3}-q_{1}\right)}{B\left(q_{1}, q_{3}-q_{1}\right)} x^{k}} .
$$


Now, consider $a_{k}^{\prime}(z)=\frac{B_{\left(s_{1}, s_{2}\right)}^{(s)}\left(q_{1}+k, z-q_{1}\right)}{B\left(q_{1}, z-q_{1}\right)}$ then, we have:

$$
\frac{\Phi_{\left(s_{1}, s_{2}\right)}^{(s)}\left(q_{1}, q_{2} ; x\right)}{\Phi_{\left(s_{1}, s_{2}\right)}^{(s)}\left(q_{1}, q_{3} ; x\right)}=\frac{\sum_{k=0}^{\infty} a_{k}^{\prime}\left(q_{2}\right) x^{k}}{\sum_{k=0}^{\infty} a_{k}^{\prime}\left(q_{3}\right) x^{k}}
$$

Now assume, $f_{k}=\frac{a_{k}^{\prime}\left(q_{2}\right)}{a_{k}^{\prime}\left(q_{3}\right)}$, then

$$
f_{k}-f_{k+1}=\frac{a_{k}^{\prime}\left(q_{2}\right)}{a_{k}^{\prime}\left(q_{3}\right)}-\frac{a_{k+1}^{\prime}\left(q_{2}\right)}{a_{k+1}^{\prime}\left(q_{3}\right)}
$$

Then, by the using above equation, we have:

$$
\begin{gathered}
f_{k}-f_{k+1}= \\
\frac{B\left(q_{1}, q_{3}-q_{1}\right)}{B\left(q_{1}, q_{2}-q_{1}\right)}\left[\frac{B_{\left(1_{1}, s_{2}\right)}^{(s)}\left(q_{1}+k, q_{2}-q_{1}\right)}{B_{\left(s_{1}, s_{2}\right)}^{(s)}\left(q_{1}+k, q_{3}-q_{1}\right)}-\frac{B_{\left(s_{1}, s_{2}\right)}^{(s)}\left(q_{1}+k+1, q_{2}-q_{1}\right)}{B_{\left(s_{1}, s_{2}\right)}^{(s)}\left(q_{1}+k+1, q_{3}-q_{1}\right)}\right] .
\end{gathered}
$$

On taking $x^{\prime}=q_{1}+n, x_{1}^{\prime}=q_{1}+k+1, y^{\prime}=q_{3}-q_{1}$ and $y_{1}^{\prime}=q_{2}-q_{1}$, we get: $\left(x^{\prime}-x_{1}^{\prime}\right) \cdot\left(y^{\prime}-y_{1}^{\prime}\right) \geq 0$ as $q_{2}-q_{3} \geq 0$

So, the above equation reduces to

$$
f_{k}-f_{k+1}=\frac{B\left(x^{\prime}-k, y^{\prime}\right)}{B\left(x^{\prime}-k, y_{1}^{\prime}\right)}\left[\frac{B_{\left(s_{1}, s_{2}\right)}^{(s)}\left(x^{\prime}, y_{1}^{\prime}\right)}{B_{\left(s_{1}, s_{2}\right)}^{(s)}\left(x^{\prime}, y^{\prime}\right)}-\frac{B_{\left(s_{1}, s_{2}\right)}^{(s)}\left(x_{1}^{\prime}, y_{1}^{\prime}\right)}{B_{\left(s_{1}, s_{2}\right)}^{(s)}\left(x_{1}^{\prime}, y^{\prime}\right)}\right] .
$$

After using the Theorem (1), we have:

$$
\frac{B_{\left(s_{1}, s_{2}\right)}^{(s)}\left(x^{\prime}, y_{1}^{\prime}\right) B_{\left(s_{1}, s_{2}\right)}^{(s)}\left(x_{1}^{\prime}, y^{\prime}\right)}{B_{\left(s_{1}, s_{2}\right)}^{(s)}\left(x_{1}^{\prime}, y_{1}^{\prime}\right) B_{\left(s_{1}, s_{2}\right)}^{(s)}\left(x^{\prime}, y^{\prime}\right)} \leq 1
$$

After re-arranging the terms, we have:

$$
\frac{B_{\left(s_{1}, s_{2}\right)}^{(s)}\left(x^{\prime}, y_{1}^{\prime}\right)}{B_{\left(s_{1}, s_{2}\right)}^{(s)}\left(x^{\prime}, y^{\prime}\right)}-\frac{B_{\left(s_{1}, s_{2}\right)}^{(s)}\left(x_{1}^{\prime}, y_{1}^{\prime}\right)}{B_{\left(s_{1}, s_{2}\right)}^{(s)}\left(x_{1}^{\prime}, y^{\prime}\right)} \leq 0 .
$$

Then, using the above result in Equation (16), we have:

$$
f_{k}-f_{k+1} \leq 0 .
$$

Which implies $f_{k} \leq f_{k+1}$ that means $\left\{f_{k}\right\}_{k \geq 0}$ is non-decreasing, non-constant sequence, i.e., increasing sequence. After the use of Lemma (1), we conclude that $x \mapsto \frac{\Phi_{\left(s_{1}, s_{2}\right)}^{(s)}\left(q_{1}, q_{2} ; x\right)}{\Phi_{\left(s_{1}, s_{2}\right)}^{(s)}\left(q_{1}, q_{3} ; x\right)}$ is increasing on $(0, \infty)$. Hence the proof of Theorem (4), is completed.

Theorem 5. For $q_{2}-q_{3} \geq 0$ the extended confluent hypergeometric function $\Phi_{\left(s_{1}, s_{2}\right)}^{(s)}\left(q_{1}, q_{2} ; x\right)$ satisfied the inequality

$$
\begin{aligned}
q_{3} \Phi_{\left(s_{1}, s_{2}\right)}^{(s)}\left(q_{1}+1, q_{2}+1 ; x\right) \Phi_{\left(s_{1}, s_{2}\right)}^{(s)}\left(q_{1}, q_{3} ; x\right) \geq & \\
& q_{2} \Phi_{\left(s_{1}, s_{2}\right)}^{(s)}\left(q_{1}+1, q_{3}+1 ; x\right) \Phi_{\left(s_{1}, s_{2}\right)}^{(s)}\left(s_{1}, s_{2} ; x\right) .
\end{aligned}
$$


Proof of Theorem 5. From the previous Theorem (4), we know that $x \mapsto \frac{\Phi_{\left(s_{1}, s_{2}\right)}^{(s)}\left(q_{1}, q_{2} ; x\right)}{\Phi_{\left(s_{1}, s_{2}\right)}^{(s)}\left(q_{1}, q_{3} ; x\right)}$ is increasing on $(0, \infty)$ for $q_{2}-q_{3} \geq 0$, then by the increasing property of the function, we have:

$$
\frac{d}{d x}\left[\frac{\Phi_{\left(s_{1}, s_{2}\right)}^{(s)}\left(q_{1}, q_{2} ; x\right)}{\Phi_{\left(s_{1}, s_{2}\right)}^{(s)}\left(q_{1}, q_{3} ; x\right)}\right] \geq 0
$$

on $(0, \infty)$.

After differentiating the function $\frac{\Phi_{\left(s_{1}, s_{2}\right)}^{(s)}\left(q_{1}, q_{2} ; x\right)}{\Phi_{\left(s_{1}, s_{2}\right)}^{(s)}\left(q_{1}, q_{3} ; x\right)}$ w.r.t $x$, we get:

$$
\begin{gathered}
\frac{\Phi_{\left(s_{1}, s_{2}\right)}^{(s)}\left(q_{1}, q_{3} ; x\right)\left(\frac{q_{1}}{q_{2}}\right) \Phi_{\left(s_{1}, s_{2}\right)}^{(s)}\left(q_{1}+1, q_{2}+1 ; x\right)}{\left[\Phi_{\left(s_{1}, s_{2}\right)}^{(s)}\left(q_{1}, q_{3} ; x\right)\right]^{2}}- \\
\frac{\left(\frac{q_{1}}{q_{3}}\right) \Phi_{\left(s_{1}, s_{2}\right)}^{(s)}\left(q_{1}+1, q_{3}+1 ; x\right) \Phi_{\left(s_{1}, s_{2}\right)}^{(s)}\left(q_{1}, q_{2} ; x\right)}{\left[\Phi_{\left(s_{1}, s_{2}\right)}^{(s)}\left(q_{1}, q_{3} ; x\right)\right]^{2}} \geq 0 .
\end{gathered}
$$

On some computation, we get our desired result.

$$
q_{3} \Phi_{\left(s_{1}, s_{2}\right)}^{(s)}\left(q_{1}+1, q_{2}+1 ; x\right) \Phi_{\left(s_{1}, s_{2}\right)}^{(s)}\left(q_{1}, q_{3} ; x\right) \geq q_{2} \Phi_{\left(s_{1}, s_{2}\right)}^{(s)}\left(q_{1}+1, q_{3}+1 ; x\right) \Phi_{\left(s_{1}, s_{2}\right)}^{(s)}\left(q_{1}, q_{2} ; x\right) .
$$

Hence proof of Theorem (5), is completed.

Theorem 6. For $\beta \in[0,1], x^{\prime}, y^{\prime}>0$, and $q_{1}, q_{2}>0$ the mapping

$$
x^{\prime} \mapsto \Phi_{\left(s_{1}, s_{2}\right)}^{(s)}\left(q_{1}, q_{2} ; x^{\prime}\right)
$$

is logarithmically convex on $(-\infty, \infty)$ and the extended confluent hypergeometric function $\Phi_{\left(s_{1}, s_{2}\right)}^{(s)}\left(q_{1}, q_{2} ; x^{\prime}\right)$ satisfies the inequalities

$$
\Phi_{\left(s_{1}, s_{2}\right)}^{(s)}\left(q_{1}, q_{2} ; \beta x^{\prime}+(1-\beta) y^{\prime}\right) \leq\left[\Phi_{\left(s_{1}, s_{2}\right)}^{(s)}\left(q_{1}, q_{2} ; x^{\prime}\right)\right]^{\beta}\left[\Phi_{\left(s_{1}, s_{2}\right)}^{(s)}\left(q_{1}, q_{2} ; x^{\prime}\right)\right]^{(1-\beta)} .
$$

Proof of Theorem 6. By the integral representation of extended hypergeometric function (5), we have:

$$
\begin{aligned}
& \Phi_{\left(s_{1}, s_{2}\right)}^{(s)}\left(q_{1}, q_{2} ; \beta x^{\prime}+(1-\beta) y^{\prime}\right)= \\
& \quad \frac{1}{B\left(q_{1}, q_{2}-q_{1}\right)} \int_{0}^{1} t^{q_{1}-1}(1-t)^{q_{2}-q_{1}-1} e^{\left(\beta x^{\prime}+(1-\beta) y^{\prime}\right) t} E_{s_{1}, s_{2}}\left(-s(t(1-t))^{-1}\right) d t .
\end{aligned}
$$

After re-arranging the terms, we get:

$$
\begin{aligned}
& \Phi_{\left(s_{1}, s_{2}\right)}^{(s)}\left(q_{1}, q_{2} ; \beta x^{\prime}+(1-\beta) y^{\prime}\right)= \\
& \frac{1}{B\left(q_{1}, q_{2}-q_{1}\right)} \int_{0}^{1}\left[\left(t^{q_{1}-1}(1-t)^{q_{2}-q_{1}-1} e^{x^{\prime} t} E_{s_{1}, s_{2}}\left(-s(t(1-t))^{-1}\right)\right)^{\beta} \times\right. \\
& \left.\quad \times\left(t^{q_{1}-1}(1-t)^{q_{2}-q_{1}-1} e^{y^{\prime} t} E_{s_{1}, s_{2}}\left(-s(t(1-t))^{-1}\right)\right)^{(1-\beta)}\right] d t .
\end{aligned}
$$


Then, from Lemma (2), we have:

$$
\begin{aligned}
& \Phi_{\left(s_{1}, s_{2}\right)}^{(s)}\left(s_{1}, s_{2} ; \beta x^{\prime}+(1-\beta) y^{\prime}\right) \leq \\
& \quad\left[\frac{1}{B\left(q_{1}, q_{2}-q_{1}\right)} \int_{0}^{1} t^{q_{1}-1}(1-t)^{q_{2}-q_{1}-1} e^{x^{\prime} t} E_{s_{1}, s_{2}}\left(-s(t(1-t))^{-1}\right) d t\right]^{\beta} \times \\
& \times\left[\frac{1}{B\left(q_{1}, q_{2}-q_{1}\right)} \int_{0}^{1} t^{q_{1}-1}(1-t)^{q_{2}-q_{1}-1} e^{y^{\prime} t} E_{s_{1}, s_{2}}\left(-s(t(1-t))^{-1}\right) d t\right]^{(1-\beta)} .
\end{aligned}
$$

After using the integral representation of the extended confluent hypergeometric function (5), we get our desired result and from above observations, we conclude that the extended confluent hypergeometric function is logarithmically convex on $(-\infty, \infty)$

$$
\Phi_{\left(s_{1}, s_{2}\right)}^{(s)}\left(q_{1}, q_{2} ; \beta x^{\prime}+(1-\beta) y^{\prime}\right) \leq\left[\Phi_{\left(s_{1}, s_{2}\right)}^{(s)}\left(q_{1}, q_{2} ; x^{\prime}\right)\right]^{\beta}\left[\Phi_{\left(s_{1}, s_{2}\right)}^{(s)}\left(q_{1}, q_{2} ; x^{\prime}\right)\right]^{(1-\beta)} .
$$

Hence, the Theorem (6), is proved.

Theorem 7. For $x, q_{1} q_{2}>0$, then the mapping

$$
q_{1} \mapsto \frac{B\left(q_{1}+q, q_{2}-q_{1}-q\right) \Phi_{\left(s_{1}, s_{2}\right)}^{(s)}\left(q_{1}+q, q_{2} ; x\right)}{B\left(q_{1}, q_{2}-q_{1}\right) \Phi_{\left(s_{1}, s_{2}\right)}^{(s)}\left(q_{1}, q_{2} ; x\right)}
$$

is decreasing function on $(0, \infty)$.

Proof of Theorem 7. Consider $q_{1}^{\prime} \geq q_{1}$,

$$
\begin{gathered}
h_{1}^{\prime}(t)=t^{q_{1}^{\prime}-1}(1-t)^{q_{2}-q_{1}^{\prime}-1} e^{x t} E_{s_{1}, s_{2}}\left(-s(t(1-t))^{-1}\right) \\
f_{1}^{\prime}(t)=\left(\frac{t}{1-t}\right)^{q_{1}-q_{1}^{\prime}} \text { and } g_{1}^{\prime}(t)=\left(\frac{t}{1-t}\right)^{q}
\end{gathered}
$$

Then, from integral representation of the extended confluent hypergeometric function (5), we have:

$$
\begin{aligned}
& \Phi_{\left(s_{1}, s_{2}\right)}^{(s)}\left(q_{1}, q_{2} ; x\right)= \\
& \frac{1}{B\left(q_{1}, q_{2}-q_{1}\right)} \int_{0}^{1} t^{q_{1}-1}(1-t)^{q_{2}-q_{1}-1} e^{x t} E_{s_{1}, s_{2}}\left(-s(t(1-t))^{-1}\right) d t .
\end{aligned}
$$

After using the above definition, we have:

$$
\begin{aligned}
& \frac{B\left(q_{1}+q, q_{2}-q_{1}-q\right) \Phi_{\left(s_{1}, s_{2}\right)}^{(s)}\left(q_{1}+q, q_{2} ; x\right)}{B\left(q_{1}, q_{2}-q_{1}\right) \Phi_{\left(s_{1}, s_{2}\right)}^{(s)}\left(q_{1}, q_{2} ; x\right)}- \\
& \frac{B\left(q_{1}^{\prime}+q, q_{2}-q_{1}^{\prime}-q\right) \Phi_{\left(s_{1}, s_{2}\right)}^{(s)}\left(q_{1}^{\prime}+q, q_{2} ; x\right)}{B\left(q_{1}^{\prime}, q_{2}-q_{1}^{\prime}\right) \Phi_{\left(s_{1}, s_{2}\right)}^{(s)}\left(q_{1}^{\prime}, q_{2} ; x\right)}= \\
& \frac{\int_{0}^{1} f_{1}^{\prime}(t) g_{1}^{\prime}(t) h_{1}^{\prime}(t) d t}{\int_{0}^{1} f_{1}^{\prime}(t) h_{1}^{\prime}(t) d t}-\frac{\int_{0}^{1} g_{1}^{\prime}(t) h_{1}^{\prime}(t) d t}{\int_{0}^{1} h_{1}^{\prime}(t) d t} .
\end{aligned}
$$

For the conditions $q \geq 0$ and $q_{1}^{\prime}-q_{1} \geq 0$, we can easily determine that the function $f_{1}^{\prime}(t)$ is decreasing and the function $g_{1}^{\prime}(t)$ is increasing, since function $h_{1}^{\prime}(t)$ is a non-negative mapping for $t \in[0,1]$. 
After by the using Lemma (3), we have:

$$
\int_{0}^{1} h_{1}^{\prime}(t) f_{1}^{\prime}(t) d t \cdot \int_{0}^{1} g_{1}^{\prime}(t) h_{1}^{\prime}(t) d t \leq \int_{0}^{1} h_{1}^{\prime}(t) d t . \int_{0}^{1} h_{1}^{\prime}(t) f_{1}^{\prime}(t) g_{1}^{\prime}(t) d t
$$

Then, after re-arranging the terms, we have:

$$
\frac{\int_{0}^{1} f_{1}^{\prime}(t) g_{1}^{\prime}(t) h_{1}^{\prime}(t) d t}{\int_{0}^{1} f_{1}^{\prime}(t) h_{1}^{\prime}(t) d t} \geq \frac{\int_{0}^{1} g_{1}^{\prime}(t) h_{1}^{\prime}(t) d t}{\int_{0}^{1} h_{1}^{\prime}(t) d t} .
$$

On some more calculations, we get:

$$
\frac{\int_{0}^{1} f_{1}^{\prime}(t) g_{1}^{\prime}(t) h_{1}^{\prime}(t) d t}{\int_{0}^{1} f_{1}^{\prime}(t) h_{1}^{\prime}(t) d t}-\frac{\int_{0}^{1} g_{1}^{\prime}(t) h_{1}^{\prime}(t) d t}{\int_{0}^{1} h_{1}^{\prime}(t) d t} \geq 0
$$

On using above relation in Equation (31), we have:

$$
\begin{gathered}
\frac{B\left(q_{1}+q, q_{2}-q_{1}-q\right) \Phi_{\left(s_{1}, s_{2}\right)}^{(s)}\left(q_{1}+q, q_{2} ; x\right)}{B\left(q_{1}, q_{2}-q_{1}\right) \Phi_{\left(s_{1}, s_{2}\right)}^{(s)}\left(q_{1}, q_{2} ; x\right)}- \\
\frac{B\left(q_{1}^{\prime}+q_{1}, q_{2}-q_{1}^{\prime}-q\right) \Phi_{\left(s_{1}, s_{2}\right)}^{(s)}\left(q_{1}^{\prime}+q, q_{2} ; x\right)}{B\left(q_{1}^{\prime}, q_{2}-q_{1}^{\prime}\right) \Phi_{\left(s_{1}, s_{2}\right)}^{(s)}\left(q_{1}^{\prime}, q_{2} ; x\right)} \geq 0 .
\end{gathered}
$$

After some calculations, we get:

$$
\begin{aligned}
& \frac{B\left(q_{1}+q, q_{2}-q_{1}-q\right) \Phi_{\left(s_{1}, s_{2}\right)}^{(s)}\left(q_{1}+q, q_{2} ; x\right)}{B\left(q_{1}, q_{2}-q_{1}\right) \Phi_{\left(s_{1}, s_{2}\right)}^{(s)}\left(q_{1}, q_{2} ; x\right)} \geq \\
& \frac{B\left(q_{1}^{\prime}+q, q_{2}-q_{1}^{\prime}-q\right) \Phi_{\left(s_{1}, s_{2}\right)}^{(s)}\left(q_{1}^{\prime}+q, q_{2} ; x\right)}{B\left(q_{1}^{\prime}, q_{2}-q_{1}^{\prime}\right) \Phi_{\left(s_{1}, s_{2}\right)}^{(s)}\left(q_{1}^{\prime}, q_{2} ; x\right)} .
\end{aligned}
$$

As $q_{1}^{\prime} \geq q_{1}$, we conclude that the mapping $q_{1} \mapsto \frac{B\left(q_{1}+q, q_{2}-q_{1}-q\right) \Phi_{\left(q_{1}, q_{2}\right)}^{(s)}\left(q_{1}+q, q_{2} ; x\right)}{B\left(q_{1}, q_{2}-q_{1}\right) \Phi_{\left(q_{1}, q_{2}\right)}^{(r)}\left(q_{1}, q_{2} ; x\right)}$ is decreasing function on $(0, \infty)$.

Hence, proof of Theorem (7), is completed.

Remark 1. In particular, the following decreasing property of the function

$$
q_{1} \mapsto \frac{B\left(q_{1}+q, q_{2}-q_{1}-q\right) \Phi_{\left(s_{1}, s_{2}\right)}^{(s)}\left(q_{1}+q, q_{2} ; x\right)}{B\left(q_{1}, q_{2}-q_{1}\right) \Phi_{\left(s_{1}, s_{2}\right)}^{(s)}\left(q_{1}, q_{2} ; x\right)}
$$

is equivalent to the inequality

$$
\begin{aligned}
& {\left[\Phi_{\left(s_{1}, s_{2}\right)}^{(s)}\left(q_{1}+q, q_{2} ; x\right)\right]^{2} \geq} \\
& \frac{B\left(q_{1}+2 q, q_{2}-q_{1}-2 q\right) B\left(q_{1}, q_{2}-q_{1}\right) \Phi_{\left(s_{1}, s_{2}\right)}^{(s)}\left(q_{1}+2 q, q_{2} ; x\right) \Phi_{\left(s_{1}, s_{2}\right)}^{(s)}\left(q_{1}, q_{2} ; x\right)}{\left[B\left(q_{1}+q, q_{2}-q_{1}-q\right)\right]^{2}} .
\end{aligned}
$$


2.2. Inequalities of Extended Gauss Hypergeometric Function $F_{\left(s_{1}, s_{2}\right)}^{(s)}\left(q_{0}, q_{1}, q_{2} ; x\right)$

Theorem 8. Let $q_{1} \geq 0$ and $q_{2}, q_{3}>0$, then for $q_{2}-q_{3} \geq 0$, the mapping

$$
x \mapsto \frac{F_{\left(s_{1}, s_{2}\right)}^{(s)}\left(q_{0}, q_{1}, q_{2} ; x\right)}{F_{\left(s_{1}, s_{2}\right)}^{(s)}\left(q_{0}, q_{1}, q_{3} ; x\right)}
$$

is increasing on $(0,1)$.

Proof of Theorem 8. By the similar procedure as used in the proof of Theorem (4), with some computation, we get our desired result of Theorem (8).

Theorem 9. For $q_{2}-q_{3} \geq 0$, the extended Gauss hypergeometric function $F_{\left(s_{1}, s_{2}\right)}^{(s)}\left(q_{0}, q_{1}, q_{2} ; x\right)$ satisfied the inequality

$$
\begin{gathered}
q_{3} F_{\left(s_{1}, s_{2}\right)}^{(s)}\left(q_{0}+1, q_{1}+1, q_{2}+1 ; x\right) F_{\left(s_{1}, s_{2}\right)}^{(s)}\left(q_{0}, q_{1}, q_{3} ; x\right) \geq \\
q_{2} F_{\left(s_{1}, s_{2}\right)}^{(s)}\left(q_{0}+1, q_{1}+1, q_{3}+1 ; x\right) F_{\left(s_{1}, s_{2}\right)}^{(s)}\left(q_{0}, q_{1}, q_{2} ; x\right) .
\end{gathered}
$$

Proof of Theorem 9. On the same parallel lines as used in the proof of Theorem (5), after some computation, we get our desired result of Theorem (9).

Theorem 10. For $x \in(0,1)$, the mapping

$$
q_{0} \mapsto F_{\left(s_{1}, s_{2}\right)}^{(s)}\left(q_{0}, q_{1}, q_{2} ; x\right)
$$

is logarithmically convex on $(0, \infty)$

Proof of Theorem 10. To prove above result, applying Theorem (3), to the extended Gauss hypergeometric function $F_{\left(s_{1}, s_{2}\right)}^{(s)}\left(q_{0}, q_{1}, q_{2} ; x\right)$.

From definition of the extended Gauss hypergeometric function (2) we have:

$$
F_{\left(s_{1}, s_{2}\right)}^{(s)}\left(q_{0}, q_{1}, q_{2} ; z\right)=\sum_{k=0}^{\infty} \frac{B_{\left(s_{1}, s_{2}\right)}^{(s)}\left(q_{1}+k, q_{2}-p_{1}\right)}{B\left(q_{1}, q_{2}-q_{1}\right)}\left(q_{0}\right) \frac{z_{k}^{k}}{k !}
$$

Now consider $f_{k}^{\prime}=\frac{B_{\left(s_{1}, s_{2}\right)}^{(s)}\left(q_{1}+k, q_{2}-q_{1}\right)}{B\left(q_{1}, q_{2}\right)}$, to prove our result it sufficient to show that the sequence $d_{k}=\left(\frac{f_{k}^{\prime}}{f_{k-1}^{\prime}}\right)$ is increasing. Clearly,

$$
d_{k}-d_{k-1}=\frac{B_{\left(s_{1}, s_{2}\right)}^{(s)}\left(q_{1}+k, q_{2}-q_{1}\right)}{B_{\left(s_{1}, s_{2}\right)}^{(s)}\left(q_{1}+k-1, q_{2}-q_{1}\right)}-\frac{B_{\left(s_{1}, s_{2}\right)}^{(s)}\left(q_{1}+k-1, q_{2}-q_{1}\right)}{B_{\left(s_{1}, s_{2}\right)}^{(s)}\left(q_{1}+k-2, q_{2}-q_{1}\right)}
$$

On using the Theorem (2), and letting $x_{1}^{\prime}=q_{1}+k, x_{2}^{\prime}=q_{1}+k-2, y_{1}^{\prime}=y_{2}^{\prime}=q_{2}-q_{1}$, we have:

$$
\begin{aligned}
& {\left[B_{\left(s_{1}, s_{2}\right)}^{(s)}\left(q_{1}+k-1, q_{2}-q_{1}\right)\right]^{2}} \\
& \quad \leq B_{\left(s_{1}, s_{2}\right)}^{(s)}\left(q_{1}+k, q_{2}-q_{1}\right) \cdot B_{\left(s_{1}, s_{2}\right)}^{(s)}\left(q_{1}+k-2, q_{2}-q_{1}\right) .
\end{aligned}
$$

After re-arranging the terms, we get:

$$
\frac{B_{\left(s_{1}, s_{2}\right)}^{(s)}\left(q_{1}+k, q_{2}-q_{1}\right)}{B_{\left(s_{1}, s_{2}\right)}^{(s)}\left(q_{1}+k-1, q_{2}-q_{1}\right)} \geq \frac{B_{\left(s_{1}, s_{2}\right)}^{(s)}\left(q_{1}+k-1, q_{2}-q_{1}\right)}{B_{\left(s_{1}, s_{2}\right)}^{(s)}\left(q_{1}+k-2, q_{2}-q_{1}\right)} .
$$


Then, by using above result in Equation (39), we have: $d_{k} \geq d_{k-1}$, which implies $\left(d_{k}\right)$ sequence is increasing. Now, we conclude by the using Theorem (3), that the mapping $q_{0} \mapsto$ $F_{\left(s_{1}, s_{2}\right)}^{(s)}\left(q_{0}, q_{1}, q_{2} ; x\right)$ is logarithmically convex on $(0, \infty)$. Hence, proof of the Theorem $(10)$, is completed.

\section{Concluding Remark}

We conclude our investigation by remarking that here, we describe some (presumably) new inequalities including the extended type Gauss hypergeometric function and confluent hypergeometric function, respectively. These inequalities are important for the approximation of extended confluent hypergeometric function, extended Gauss hypergeometric function, generalized Appell and Lauricella hypergeometric functions. We hope our investigation is capable of providing potential directions for future research in the approximation theory and applications of special functions.

Author Contributions: Conceptualization, S.J. and R.G.; methodology, P.A.; software, S.J. and R.G.; validation, S.J., R.G., P.A. and J.L.G.G.; investigation, J.L.G.G.; formal analysis, P.A. and J.L.G.G.; resources, P.A.; data writing—original draft preparation, S.J.; writing—review and editing, S.J., R.G., P.A. and J.L.G.G.; visualization, P.A.; funding acquisition, P.A. and J.L.G.G. All authors have read and agreed to the published version of the manuscript.

Funding: The third author is partially supported by Ministerio de Ciencia, Innovación y Universidades grant number PGC2018-097198-B-I00 and Fundación Séneca de la Región de Murcia grant number 20783/PI/18.

Acknowledgments: Juan L. G. Guirao is thankful to the Ministerio de Ciencia, Innovación y Universidades grant number PGC2018-097198-B-I00 and Fundación Séneca de la Región de Murcia grant number 20783/PI/18 and Shilpi Jain thankful to SERB (project number: MTR/2017/000194) for providing necessary facilities during this research work.

Conflicts of Interest: The authors declare no conflict of interest.

\section{References}

1. Rainville, E.D. Special Functions; Macmillan: New York, NY, USA, 1960.

2. Olver, W.J.F.; Lozier, W.D.; Boisvert, F.R.; Clark, W.C. NIST Handbook of Mathematical Functions; Cambridge University Press: New York, NY, USA, 2010.

3. Agarwal, P.; Dragomir, S.S.; Jleli, M.; Samet, B. (Eds.) Advances in Mathematical Inequalities and Applications; Springer: Berlin, Germany, 2018.

4. Luo, M.J.; Milovanovic, G.V.; Agarwal, P. Some results on the extended beta and extended hypergeometric functions. Commun. Appl. Math. Comput. 2014, 248, 631-651. [CrossRef]

5. Butt, S.I.; Pečarić, J.; Rehman, A.U. Exponential convexity of Petrović and related functional. J. Inequal. Appl. 2011, $2011,1-6$. [CrossRef]

6. Qi, F.; Li, L.H.; Xu, S.L. Some inequalities constructed by Tchebysheff's integral inequality. Math. Inequal. Appl. 1999, 2, 517-528. [CrossRef]

7. Gorenflo, R.; Kilbas, A.A.; Mainardi, F.; Rogosin, S.V. Mittage Leffler Functions Related Topics and Application, 2nd ed.; Springer: New York, NY, USA, 2020.

8. Wiman, A. Über die Nullstellun der Funktionen $E_{\alpha}(x)$. Acta Math. 1905, 29, 217-234. [CrossRef]

9. Goyal, R.; Agarwal, P.; Momami, S.; Rassias, M.T. An Extension of Beta Function by Using Wiman's function. Axioms 2021, 10, 187. [CrossRef]

10. Jain, S.; Goyal, R.; Agarwal, P.; Lupica, A.; Cesarano, C. Some results of extended beta function and hypergeometric functions by using Wiman's function. 2021, submitted-under review.

11. Biernacki, M.; Krzyż, J. On the monotonity of certain functionals in the theory of analytic functions. Ann. Univ. Mariae-CurieSkłodowska Sect. A 1955, 9, 135-147.

12. Rudin, W. Real and Complex Analysis, 3rd ed.; McGraw-Hill Book Co.: New York, NY, USA, 1987.

13. Tian, J.F.; Ha, M.H. Properties of generalized sharp Hölder's inequalities. J. Math. Inequal. 2017, 11, 511-525. [CrossRef]

14. Qi, F.; Li, W.H. logarithmically completely monotonic function involving the ratio of gamma functions. J. Appl. Anal. Comput. 2015, 5, 626-634.

15. Dragomir, S.S.; Agarwal, R.P.; Barnett, N.S. Inequalities for Beta and Gamma functions via some classical and new integral inequalities. J. Inequal. Appl. 2000, 5, 103-165. [CrossRef] 
16. Kumar, P.; Singh, S.P.; Dragomir, S.S. Some inequalities involving beta and gamma functions, Nonlinear analysis and its applications. Nonlinear Anal. Forum. 2001, 6, 143-150.

17. Karp, D.; Sitnik, S.M. Log-convexity and log-concavity of hypergeometric-like functions. J. Math. Anal. Appl. 2010, 364, 384-394. [CrossRef] 